\title{
Elimination of Redundant Synaptic Inputs in the Absence of Synaptic Strengthening
}

\author{
Hao Wang, Hong Liu, and Zhong-wei Zhang \\ The Jackson Laboratory, Bar Harbor, Maine 04609
}

Synaptic refinement, a developmental process that consists of selective elimination and strengthening of immature synapses, is essential for the formation of precise neuronal circuits and proper brain function. At glutamatergic synapses in the brain, activity-dependent recruitment of AMPA receptors (AMPARs) is a key mechanism underlying the strengthening of immature synapses. Studies using receptor overexpression have shown that the recruitment of AMPARs is subunit specific. With the notable exception of hippocampal CA3-CA1 synapses, however, little is known about how native receptors behave or the roles of specific AMPAR subunits in synaptic refinement in vivo. Using patch-clamp recordings in acute slices, we examined developmental refinement of whisker relay (lemniscal) synapses in the thalamus in mice deficient of AMPAR subunits. Deletion of GluA3 or GluA4 caused significant reductions of synaptic AMPAR currents in thalamic neurons at P16-P17, with a greater reduction observed in GluA3-deficient mice. Deletions of both GluA3 and GluA4 abolished synaptic AMPAR responses in the majority of thalamic neurons, indicating that at thalamic relay synapses AMPARs are composed primarily of GluA3 and GluA4. Surprisingly, deletions of GluA3 or GluA4 or both had no effect on the elimination of relay inputs: the majority of thalamic neurons in these knock-out mice-as in wild-type mice—receive a single relay input. However, experience-dependent strengthening of thalamic relay synapses was impaired in GluA3 knock-out mice. Together these findings suggest that the elimination of immature glutamatergic synapses proceeds normally in the absence of synaptic strengthening, and highlight the role of GluA3-containing AMPARs in experience-dependent synaptic plasticity.

\section{Introduction}

Refinement of synaptic connections is a key step in the formation of neuronal circuits (Lichtman and Colman, 2000; Luo and O'Leary, 2005; Kano and Hashimoto, 2009). The removal of redundant inputs (synapse elimination) and strengthening of the remaining ones are the two essential aspects of the refinement process. At the neuromuscular junction, strengthening of synapses precedes the removal of redundant inputs (Colman et al., 1997); a similar phenomenon was observed in the cerebellum (Hashimoto and Kano, 2003). At sensory relay synapses in the thalamus, synaptic strengthening and elimination of redundant inputs occur during the same period (Chen and Regehr, 2000; Arsenault and Zhang, 2006; Zhang, 2006). Despite the temporal correlation, it is unclear whether these two aspects of refinement are mechanistically related. At the neuromuscular junction, clustering of acetylcholine (ACh) receptors at the postsynaptic site is a key step in synaptic strengthening (Sanes and Lichtman, 1999). Genetically silencing ACh synthesis in motor neurons leads to

\footnotetext{
Received Sept. 7, 2011; revised Sept. 27, 2011; accepted Sept. 28, 2011.

Author contributions:H.W. and Z.-w.Z. designed research;H.W., H.L., andZ.-w.Z. performed research; H.W., H.L., and Z.-w.Z. analyzed data; Z.-w.Z. wrote the paper.

This work was supported by NIH Grant NS064013 (Z.-w.Z.). We thank Wayne Frankel for kindly providing breeder pairs of the GluA3 and GluA4 knock-out mice, Rob Burgess and Da-Ting Lin for comments on a previous version of this manuscript, Wen Zhang for participating in some experiments, and Matthew Peterson for technical assistance.

Correspondence should be addressed to Dr. Zhong-wei Zhang, The Jackson Laboratory, 600 Main Street, Bar Harbor, ME 04609. E-mail: zhongwei.zhang@jax.org.

DOI:10.1523/JNEUROSCI.4569-11.2011

Copyright $\odot 2011$ the authors $\quad 0270-6474 / 11 / 3116675-10 \$ 15.00 / 0$
}

extensive branching of motor neuron axons and hyperinnervation of muscle fibers (Misgeld et al., 2002). Similar effects have been observed in mice in which ACh receptors have been deleted (An et al., 2010). These findings support the idea that signaling through postsynaptic ACh receptors inhibits the growth and branching of motor neuron axons. However, at least some of these effects appear to be mediated by ACh receptors located on cells other than the muscle (An et al., 2010).

At glutamatergic synapses in the vertebrate nervous system, upregulation of AMPA receptors (AMPARs) is a key mechanism underlying synaptic strengthening. Immature synapses contain few or no AMPARs; functional AMPARs are gradually recruited to the synapse to become the primary mediator of excitatory transmission in the developing and adult brain (Isaac et al., 1995, 1997; Wu et al., 1996; Petralia et al., 1999). Activity-dependent trafficking of AMPARs is thought to be the key mechanism underlying strengthening and plasticity of glutamatergic synapses (Malinow and Malenka, 2002; Bredt and Nicoll, 2003; Collingridge et al., 2004). Native AMPARs are heteropentamers composed of four subunits, GluA1 through GluA4 (Mayer and Armstrong, 2004). Activity-dependent trafficking of AMPARs has been implicated in long-term synaptic plasticity in the hippocampus and cerebellum (Song and Huganir, 2002; Kerchner and Nicoll, 2008). Despite the role of AMPARs in synaptic plasticity, it is not clear whether developmental upregulation of AMPARs is implicated in the elimination of redundant inputs. In addition, little is known about developmental regulation of native synaptic AMPARs in the brain. 
We attempted to answer these questions by studying the lemniscal synapse in the ventral posteromedial nucleus (VPm) of the thalamus in the mouse. Tactile information from large vibrissa is transmitted to VPm neurons by axons from the principal sensory trigeminal nucleus (PrV) in the brainstem. This pathway undergoes extensive refinement during early life (Arsenault and Zhang, 2006; Wang and Zhang, 2008). Using mice deficient of AMPAR subunits, we show that the developmental upregulation of AMPARs is not necessary for the removal of redundant synaptic inputs.

\section{Materials and Methods}

Mice. GluA3 and GluA4 knock-out mice (Gria $3^{\text {tm1Dgen }}$ and Gria $4^{\text {tm1Dgen }}$ ) were developed and initially characterized by Deltagen (see http://www. informatics.jax.org/external/ko/deltagen/1623.html; http://www.informatics. jax.org/external/ko/deltagen/1624.html). The original strains were on a mixed 129P2/OlaHsd;C57BL/6 background. These two strains were imported by Dr. Wayne Frankel from the NIH-funded Mouse Mutant Regional Resource Center (MMRRC; http://www.mmrrc.org) at University of North Carolina, and were further characterized by Dr. Frankel's group (Beyer et al., 2008). Both strains have been backcrossed to C57BL/6J (B6) for $>11$ generations. Because Gria3 is X-linked, Gria $3^{\text {tmlDgen }}$ is maintained by mating heterozygous females with B6 males, and male mutants and wildtype littermates were used in the experiments. Gria $4^{\text {tm1Dgen }}$ is maintained by heterozygous mating, and both male and female mutants were used. GluA3/4 double knock-out (DKO) mice were obtained by mating female Gria ${ }^{\text {tm1Dgen/wt}} ;$ Gria $4^{\text {tm1Dgen/tm1Dgen }}$ with male Gria $3^{\mathrm{wt} / \mathrm{y}}$; Gria4 $^{\text {tm1Dgen/tm1Dgen }}$. Neither Gria3 ${ }^{\text {tm1Dgen }}$ nor Gria4 ${ }^{\text {tm1Dgen }}$ homozygous mutants show any obvious behavioral defect. GluA3/4 DKO mice develop ataxia after 2 weeks of age, but survive to adulthood.

Genotyping was performed as described previously (Beyer et al., 2008). For Gria ${ }^{\text {tmlDgen }}$, genotypes were determined by standard PCR using three primers: 5' -GGTCACGAGGTTCTTCATTGTTGTC-3', 5' GGGTGGGATTAGATAAATGCCTGCTCT-3', and 5' -AGCTGATATA GCTGTTGCTCCACTC-3', which respectively amplify 275 and 380 bp fragments from the wild-type and mutated alleles. For Gria $4^{\text {tm1Dgen }}$, genotypes were determined by standard PCR using two primers: $5^{\prime}$ CGGTCCAATTAGTTCGGTTT-3' , and 5'-GATGGGACTCGGCTAT TGAA- $3^{\prime}$, which respectively amplify 133 and $151 \mathrm{bp}$ fragments from the wild-type and mutated alleles.

All procedures are in accordance with the NIH Guide for the Care and Use of Laboratory Animals, and have been approved by The Jackson Laboratory Animal Care and Use Committee.

Whisker deprivation. Mice aged P13 (13 d postnatal with the day of birth as P0) were anesthetized by isoflurane. Mice were placed under a dissection microscope, and large whiskers on the snout were pulled out gently with a pair of forceps. This method does not damage follicles.

Slice preparation. For recordings in the thalamus, sagittal sections 300 $\mu \mathrm{m}$ thick were prepared using methods described previously (Arsenault and Zhang, 2006). For recordings in the PrV, horizontal slices $250 \mu \mathrm{m}$ thick were prepared as we described previously (Timofeeva et al., 2005). Slices were kept in artificial CSF (ACSF) containing the following (in mм): $124 \mathrm{NaCl}, 3.0 \mathrm{KCl}, 1.5 \mathrm{CaCl}_{2}, 1.3 \mathrm{MgCl}_{2}, 1.0 \mathrm{NaH}_{2} \mathrm{PO}_{4}, 26$ $\mathrm{NaHCO}_{3}$, and 20 glucose, saturated with $95 \% \mathrm{O}_{2}$ and $5 \% \mathrm{CO}_{2}$ at room temperature $\left(21-23^{\circ} \mathrm{C}\right)$.

Patch-clamp recording. Recordings were made at $32-34^{\circ} \mathrm{C}$. Unless specified otherwise, the pipette solution contained the following (in $\mathrm{mm}$ ): 110 Cs methylsulfate, 20 TEA-Cl, $15 \mathrm{CsCl}, 4$ ATP-Mg, 0.3 GTP, 0.5 EGTA, 10 HEPES, 4.0 QX-314, and 1.0 spermine ( $\mathrm{pH} \mathrm{7.2,} \mathrm{270-280}$ mOsm with sucrose). Electrodes had resistances between 2 and $4 \mathrm{M} \Omega$. Whole-cell recordings were made at the soma of VPm neurons with a Multiclamp 700B amplifier (Molecular Devices). The series resistance $\left(R_{\mathrm{s}}\right)$, usually between 8 and $14 \mathrm{M} \Omega$, was not compensated in voltageclamp experiments. Data were discarded when $R_{\mathrm{s}}$ was $>18 \mathrm{M} \Omega$. A concentric bipolar electrode (FHC) was placed in the medial lemniscus, and stimuli (150 $\mu \mathrm{s}, 10-900 \mu \mathrm{A}$, with the center pole being negative) were applied at $0.1 \mathrm{~Hz}$. For recordings in PrV neurons, the stimulation electrode was placed in the sensory root of the trigeminal nerve. GABAergic and glycinergic transmission was blocked by $100 \mu \mathrm{M}$ picrotoxin and 2 $\mu \mathrm{M}$ strychnine in the bath. Experiments were conducted using AxoGraph X (AxoGraph Scientific). Data were filtered at $4 \mathrm{kHz}$ and digitized at $20 \mathrm{kHz}$.

In some experiments, D-APV [D-(-)-2-amino-5-phosphonopentanoic acid] was used to block NMDA receptors. D-APV (100 $\mu \mathrm{M}$ in ACSF saturated with $95 \% \mathrm{O}_{2}$ and $5 \% \mathrm{CO}_{2}$ ) was applied to the recorded neuron by gravity using glass tubing ( $250 \mu \mathrm{m}$ I.D., Polymicro Technologies). D-APV and picrotoxin were obtained from Tocris Bioscience, and all other chemicals were obtained from Sigma-Aldrich.

For current-clamp recording, the pipette solution contained the following (in mM): $120 \mathrm{~K}^{+}$-gluconate, $10 \mathrm{KCl}, 4 \mathrm{ATP}-\mathrm{Mg}, 0.3 \mathrm{GTP}, 10$ HEPES, and 0.5 EGTA ( $\mathrm{pH} 7.2,270-280 \mathrm{mOsm}$ with sucrose). Series resistance was fully compensated using the bridge circuit of the amplifier.

Data analysis. All data analyses were done without prior knowledge of genotype or treatment. The number of inputs for each VPm neuron was estimated as we described previously (Arsenault and Zhang, 2006; Wang and Zhang, 2008). EPSCs evoked from the same cell over a wide range of stimulus intensity (typically from 20 to $900 \mu \mathrm{A}, 200 \mu \mathrm{s}$ ) were analyzed to determine the number of increments in the amplitude of EPSCs. For each cell, the stimulus intensity was increased with steps of 10 or $20 \mu \mathrm{A}$. At P12-P13 and P16-P17, neurons showed 1-4 increments, all of which could be clearly identified. At P7, however, VPm neurons showed a large number of small increments, which makes increment counting unreliable. This is particular problematic for EPSC-AMPAR recorded at $-70 \mathrm{mV}$ because the amplitudes of EPSC-AMPAR are very small at P7. Therefore, we estimated the input number from EPSC-NMDAR recorded at $+40 \mathrm{mV}$. We divided the amplitude of the third increment by 3 to provide an estimation of the average single-input response. We chose the third increment because it can be easily identified in all cells at P7, and our tests showed it produces similar results as with the fifth increment (divided by 5 in this case). The maximal response of a cell was divided by the average single-input response to provide an estimation of the number of inputs for the cell. In mice deficient of GluA3 or both GluA3 and GluA4, the amplitude of EPSC-AMPAR was small at P12-P13 and P16-P17; the input number in these mice was estimated from EPSC-NMDAR recorded at $+40 \mathrm{mV}$. For wild-type and GluA4-KO mice at P12-P13 and $\mathrm{P} 16-\mathrm{P} 17$, the input number could be estimated using either EPSC-AMPAR or EPSC-NMDAR; the two methods gave the same results.

The AMPAR/NMDAR ratio was estimated by measuring EPSC amplitudes at $-70 \mathrm{mV}$ and $+40 \mathrm{mV}$ at the same stimulus intensity from the same cell. The peak amplitude at $-70 \mathrm{mV}$ was used for AMPAR-EPSC. For NMDA-EPSC at $+40 \mathrm{mV}$, amplitude at $10 \mathrm{~ms}$ after the beginning of EPSCs was measured.

Intrinsic properties were analyzed using methods that we described previously (Zhang, 2004). Briefly, resting potentials of VPm neurons were measured within $20 \mathrm{~s}$ of establishing whole-cell configuration. Input resistance was estimated from voltage responses to $400 \mathrm{~ms}$ current pulses at $-100 \mathrm{pA}$. Action potentials were evoked using positive current pulses. Action potential threshold was estimated as the point when the slope of rising membrane potential exceeds $50 \mathrm{mV} /$ $\mathrm{ms}$, and for each cell measurements of five action potentials were averaged.

AxoGraph X was used for analysis. Statistics was performed using IgorPro (WaveMetrics) and InStat (GraphPad). Throughout, means are given \pm SEM. Means were compared using one-way ANOVA or MannWhitney test. Distributions of cells receiving different number of inputs were compared using $\chi^{2}$ test.

\section{Results}

GluA3 and GluA4 are the major AMPA receptor subunits at the VPm relay synapse

The lemniscal synapse in the VPm reaches functional maturity by P16 in the mouse (Arsenault and Zhang, 2006; Wang and Zhang, 2008). At this age, synaptic transmission at resting membrane 
A
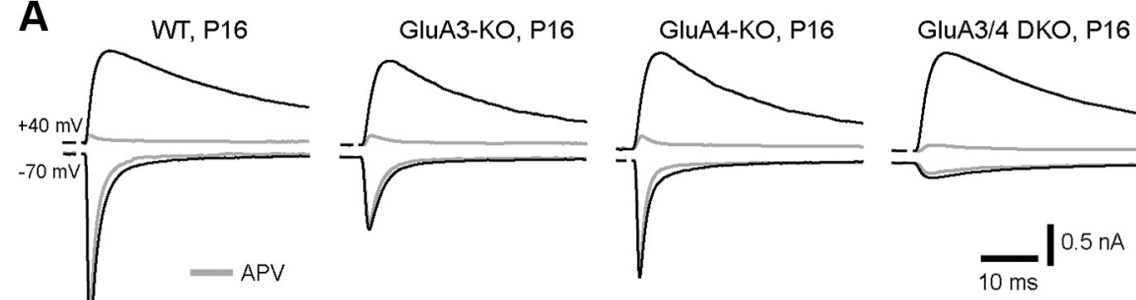

B

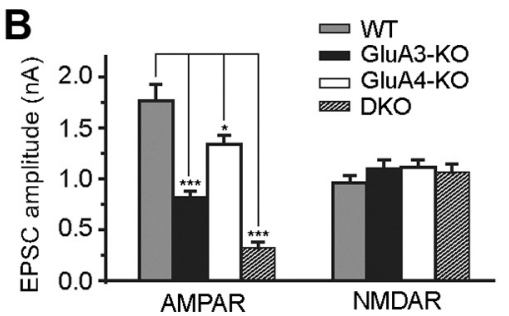

C

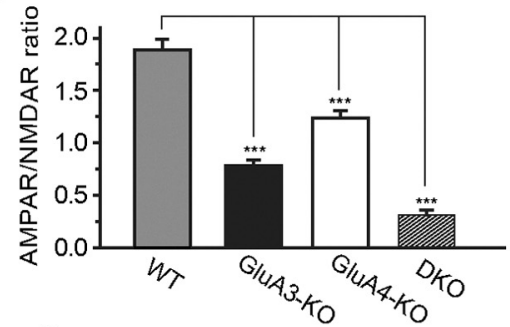

D

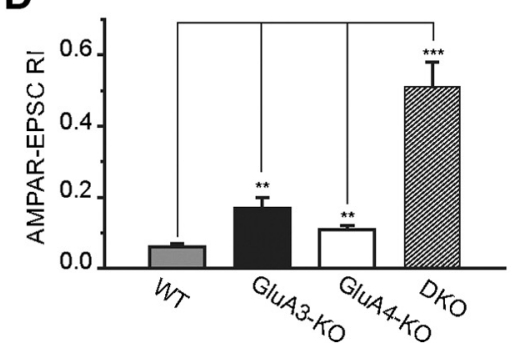

E $\underset{0}{0}$

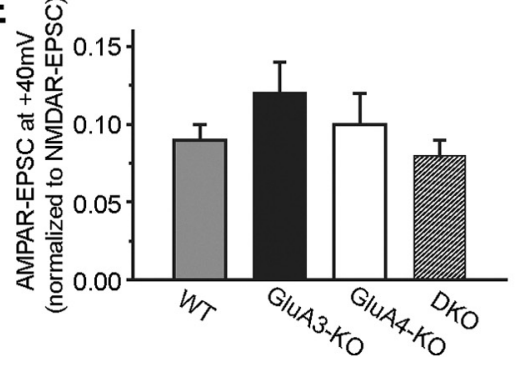

Figure 1. Reduction of AMPAR-mediated EPSCs in VPm neurons of mice deficient of GluA3, GluA4, or both. $A$, EPSCs recorded at +40 (upper traces) and $-70 \mathrm{mV}$ (lower traces) in absence (in black) or presence of D-APV (100 $\mu \mathrm{m}$; in gray) from wild-type (WT), GluA3-KO, GluA4-K0, and GluA3/4 DKO mice at P16. B, Peak amplitudes of EPSCs at -70 and $+40 \mathrm{mV}$ recorded from WT (gray), GluA3-K0 (black), GluA4-K0 (empty), and DK0 (hatch) mice at P16-P17.C, The AMPAR/NMDAR ratio in VPm neurons from the four groups at P16 -P17.D, Rectification index (RI) of AMPAR-EPSCs for the four groups. RI was calculated as the ratio of peak amplitude of AMPAR-EPSCs at $+40 \mathrm{mV}$ to that at $-70 \mathrm{mV}$ in the presence of D-APV. ${ }^{*} p<0.05,{ }^{* *} p<0.005,{ }^{* * *} p<0.0001 . E$, Normalized peak amplitudes of AMPAR-EPSCs recorded at $+40 \mathrm{mV}$ for the four groups. For each cell, the peak amplitude of AMPAR-EPSCS recorded at $+40 \mathrm{mV}$ in the presence of APV was normalized to that of NMDAR-EPSCs recorded before APV application. Normalized amplitudes were $0.09 \pm 0.01(n=8)$ for WT, $0.12 \pm 0.02(n=11)$ for GluA3-K0, $0.10 \pm 0.02(n=7)$ for GluA3-K0, and 0.08 \pm $0.01(n=10)$ for $\operatorname{DKO}(p>0.3)$.

potential is dominated by AMPAR-mediated current. However, the types of AMPAR subunits involved at this synapse were unclear. Data obtained with in situ hybridization and immunostaining have shown that GluA2, GluA3, and GluA4, but not GluA1, are expressed in the VPm, with GluA3 and GluA4 expressed at higher levels than GluA2 (Liu, 1997; Mineff and Weinberg, 2000) (Allen Brain Atlas: http://mouse.brain-map.org). This is consistent with our previous electrophysiological data indicating that VPm relay synapses contain few GluA2 subunits (Wang and Zhang, 2008). These observations led us to hypothesize that GluA3 and GluA4 are the major AMPAR subunits at the VPm relay synapse. We examined the contribution of GluA3 and GluA4 at this synapse in mice deficient of GluA3 (GluA3-KO), GluA4 (GluA4-KO), or both (DKO).

Whole-cell patch-clamp recordings were made in VPm neurons in acute brain slices prepared from P16-P17 mice as we described previously (Arsenault and Zhang, 2006). Whisker relay (lemniscal) synapses were selectively activated by stimuli applied to the medial lemniscus. We recorded the maximal synaptic responses in VPm neurons of WT, GluA3-KO, GluA4-KO, and GluA3/4 DKO mice. As illustrated in Figure $1 A$ (traces in black), peak amplitudes of EPSCs recorded at $-70 \mathrm{mV}$ were significantly reduced in mutant mice. Compared with that of wild-type mice
(Fig. $1 B ; 1768 \pm 157 \mathrm{pA} ; n=17$ ), the peak amplitude of the maximal EPSC at -70 $\mathrm{mV}$ was reduced by $54 \%$ in GluA3-KO $(811 \pm 67 \mathrm{pA}, n=21 ; p<0.0001), 24 \%$ in GluA4-KO (1335 \pm 93 pA, $n=22 ; p<$ 0.02 ), and $82 \%$ in GluA3/4 DKO (323 \pm $51 \mathrm{pA}, n=17 ; p<0.0001)$. In contrast to that at $-70 \mathrm{mV}$, the peak amplitude of the maximal EPSC at $+40 \mathrm{mV}$, measured at $10 \mathrm{~ms}$ from the rise phase, was not significant different among the four genotypes (Fig. $1 B ; p>0.2 ; 955 \pm 76 \mathrm{pA}$ for WT; $1094 \pm 88$ pA for GluA3-KO; $1114 \pm 72$ pA for GluA4-KO; $1061 \pm 80 \mathrm{pA}$ for DKO). To selectively analyze AMPA and NMDA receptor-mediated EPSCs at the synapse, we applied the selective NMDAR antagonist D-APV. Consistent with our previous results obtained from B6 mice, applications of D-APV $(100 \mu \mathrm{M})$ had no effect on peak amplitudes of EPSCs recorded at $-70 \mathrm{mV}$, but completely blocked the slow component of EPSCs recorded at $+40 \mathrm{mV}$ (Fig. $1 \mathrm{~A}$, traces in gray). We estimated the AMPAR/ NMDAR ratio from individual neurons by measuring amplitude of EPSCs (in the absence of D-APV) at -70 and $+40 \mathrm{mV}$ at the same stimulation intensity. The AMPAR/NMDAR ratio was significantly reduced in the three mutant groups (Fig. $1 C$; $p<0.0001$ ), with GluA3/4 DKO neurons showing the largest reduction (84\%). These findings indicate that GluA3 and GluA4 are the major AMPAR subunits at the VPm relay synapses, and that deletion of GluA3, GluA4, or both does not alter the function of NMDARs at the synapse.

Consistent with our previous studies in B6 mice (Wang and Zhang, 2008), AMPAR-EPSCs in WT mice showed a strong inward rectification, indicating that the majority of AMPARs at the synapse lack GluA2 subunit. We analyzed the rectification of AMPARs in WT, GluA3-KO, GluA4-KO, and GluA3/4 DKO mice by measuring EPSC peak amplitudes at -70 and $+40 \mathrm{mV}$ in the presence of D-APV. The ratio of AMPAR-EPSC at $+40 \mathrm{mV}$ to that at $-70 \mathrm{mV}$ was used as the rectification index. Deletion of GluA3 or GluA4 caused moderate increases in the rectification index, whereas deletion of both dramatically increased the rectification index (Fig. $1 D ; 0.06 \pm 0.01, n=8$ for WT; $0.17 \pm 0.03, n=11$ for GluA3$\mathrm{KO}, p<0.005 ; 0.11 \pm 0.01, n=7$ for GluA4-KO, $p<0.005$; $0.51 \pm 0.07, n=10$ for DKO, $p<0.0001)$. These results suggest that the majority of GluA3 and GluA4 at the synapse are not associated with GluA2.

To quantify the number of GluA2-containing AMPARs at the synapse, we analyzed EPSCs recorded at $+40 \mathrm{mV}$ in the presence of D-APV. The peak amplitude of AMPAR-EPSCs at $+40 \mathrm{mV}$ was normalized to that of NMDAR-EPSCs recorded at $+40 \mathrm{mV}$ before APV application. As shown in Figure 1E, no significant difference was found among the four groups in AMPAR-EPSCs at $+40 \mathrm{mV}$ ( $p>0.3$, ANOVA), suggesting that deletion of GluA3, GluA4, or both had little or no effect on the number of GluA2containing AMPARs at the VPm relay synapse. 
A

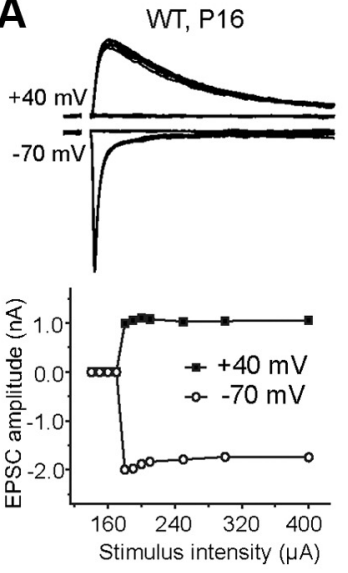

$\mathbf{E}$

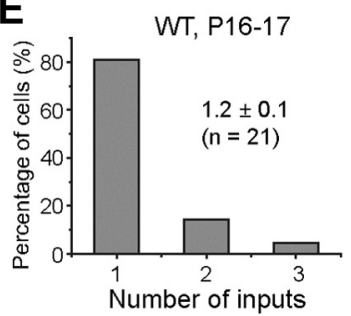

B
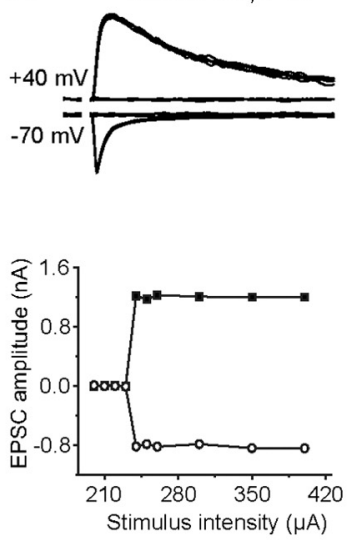

F GluA3-KO, P16-17

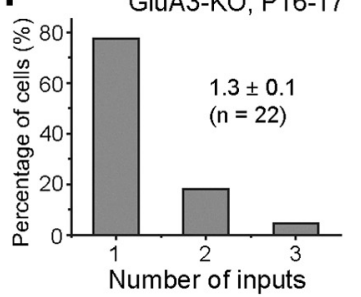

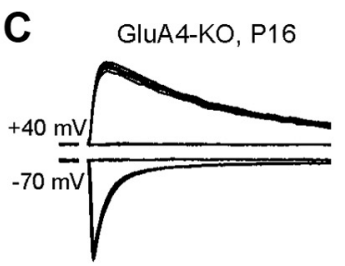
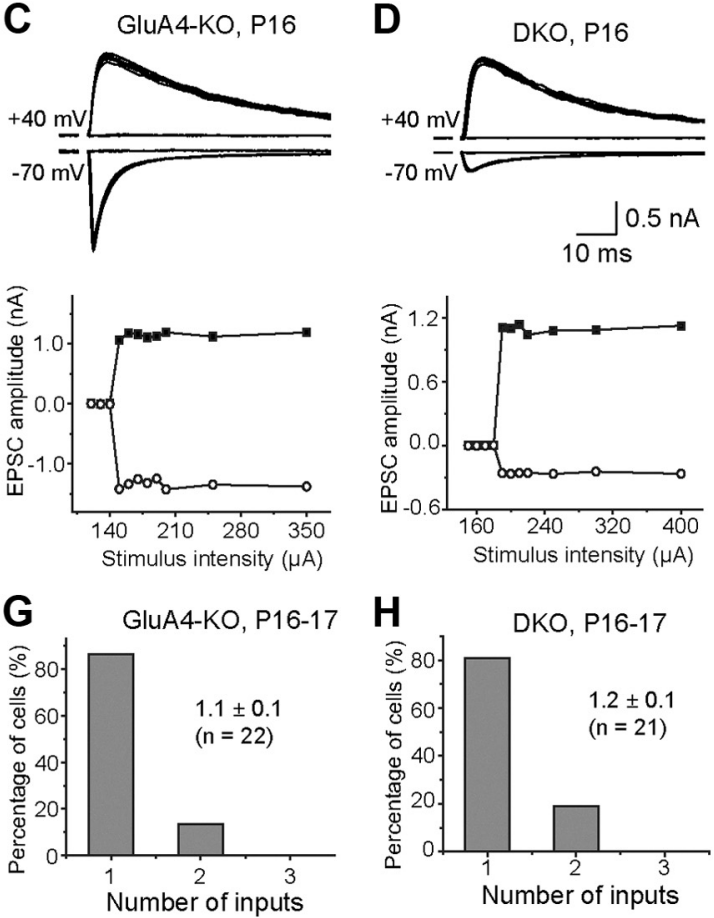

Figure 2. No change in the number of relay inputs in VPm neurons of mice deficient of GluA3, GluA4, or both at P16-P17. A, Top panel, Synaptic currents in response to a range of stimulation intensity in a VPm neuron from a WT mouse at P16; the lower panel is the plot of peak amplitude of EPSCs recorded from the cell at +40 (filled square) and $-70 \mathrm{mV}$ (open circle) versus intensity. $\boldsymbol{B}-\boldsymbol{D}$, Results obtained from GluA3-K0, GluA4-K0, and GluA3/4 DK0, respectively. $\boldsymbol{E}-\boldsymbol{H}$, Distributions of neurons at P16-P17 receiving different number of relay inputs. For all four genotype groups, about $80 \%$ of VPm neurons received a single relay input at P16 -P17. The average number of fibers per cell for each group was shown in the figures.

\section{Synapse elimination proceeds normally in mice deficient of GluA3, GluA4, or both}

The refinement of synaptic connections in nervous systems consists of elimination and strengthening of immature synapses. During development synaptic strengthening often precedes synapse elimination. To test whether strengthening of synapses regulates the process of synapse elimination, we first examined the number of relay inputs at the VPm relay synapses in wild-type, GluA3-KO, GluA4-KO, and DKO mice at P16-P17. As illustrated in Figure 2, about $80 \%$ of VPm neurons in all four genotypes showed an all-or-none response, indicating that they received a single PrV axon. The distributions of VPm neurons receiving different input numbers are not significantly different between any two genotypes ( $p>0.3, \chi^{2}$ test). Therefore, despite significant reductions in AMPAR function, the PrV-VPm pathway in the knock-out mice showed the same number of relay inputs as in wild-type mice.

To determine whether deletion of GluA3, GluA4, or both alters the pattern of connectivity at early stage of refinement, we recorded VPm neurons from wild-type, GluA3-KO, GluA4-KO, and DKO mice at P7. The majority of neurons in all four genotypes showed multiple increments in response to a range of stimulus intensity (Fig. 3). The distributions of estimated input numbers were not significantly different among the four groups. The mean input number per neuron was $6.2 \pm 0.5(n=20)$ for wild-type, $6.2 \pm 0.4(n=20)$ for GluA3-KO, $6.5 \pm 0.5(n=21)$ for GluA4-KO, and $6.7 \pm 0.4(n=21)$ for GluA3/4 DKO $(p>$ 0.3 , ANOVA).

Our previous studies showed that the period of P12-P13 is critical for activity-dependent plasticity at the VPm relay synapse (Wang and Zhang, 2008). We recorded VPm neurons from wildtype, GluA3-KO, GluA4-KO, and DKO mice at P12-P13. As illustrated in Figure 4, the distributions of neurons receiving different input numbers were not significantly different between the four groups. The mean input number per cell was $1.7 \pm 0.2$ $(n=21)$ for wild-type, $1.6 \pm 0.1(n=21)$ for GluA3-KO, $1.8 \pm$ $0.2(n=22)$ for GluA4-KO, and $1.6 \pm 0.2(n=23)$ for DKO $(p>$ 0.7, ANOVA).

Together these results indicate that despite being the major components of AMPARs at the VPm relay synapse, GluA3 and GluA4 are not involved in the process of synapse elimination in the thalamus.

The results obtained in GluA3/4 DKO mice indicate that elimination of redundant inputs proceeds normally in the VPm despite a dramatic reduction in the strength of synapses. One caveat is that the remaining AMPAR and NMDAR in DKO mice may be sufficient to generate action potential in the postsynaptic neurons. We tested this possibility with current-clamp recordings in VPm neurons at P12-P13. This age was chosen because our previous study has shown that sensory activity at P12-P13 is important for elimination of relay inputs in the VPm (Wang and Zhang, 2008). The intracellular solution contained $120 \mathrm{mM} \mathrm{K}^{+}$gluconate and $10 \mathrm{~mm} \mathrm{KCl}$. Upon establishing whole-cell configuration, VPm neurons were voltage clamped at $-70 \mathrm{mV}$ to record evoked EPSCs in response to stimuli applied to the medial lemniscus (Fig. 5A, $B$, upper traces). Then synaptically evoked responses were recorded in current-clamp mode (Fig. 5A, $B$, lower traces). At intensity that produced the maximal EPSCs, stimulation of relay inputs caused postsynaptic action potential in $90 \%$ wild-type neurons ( 19 of 21 cells from two mice), whereas only $22 \%$ of mutant cells ( 4 of 18 cells from two DKO mice) showed synaptically evoked action potential $(p<0.0001$, Fisher's exact test). We also analyze intrinsic properties of VPm neurons in current clamp, and found no difference between wild-type and mutant neurons in the resting membrane potential $(-59.2 \pm 0.6$ vs $-58.4 \pm 0.6 \mathrm{mV} ; p>0.3)$, cell input resistance $(145.9 \pm 8.3 \mathrm{vs}$ 
A
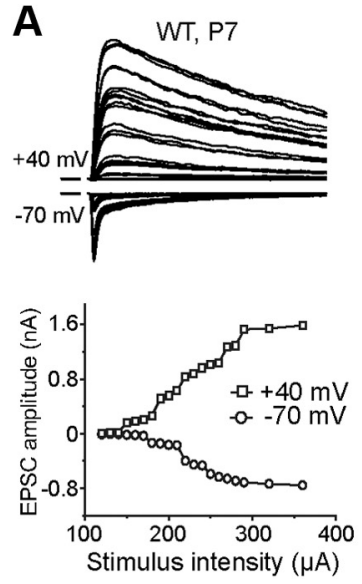

$\mathbf{E}$

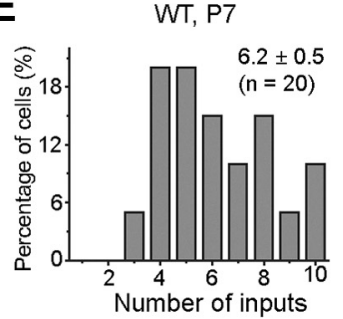

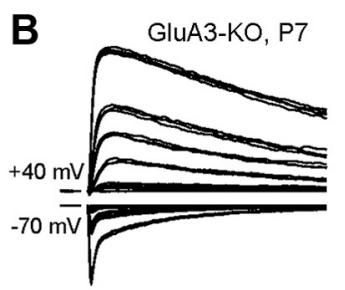

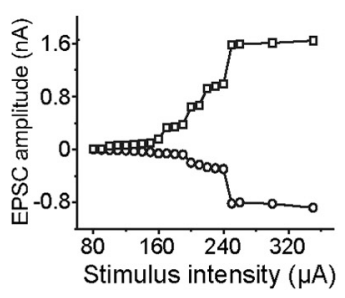

$\mathbf{F}$

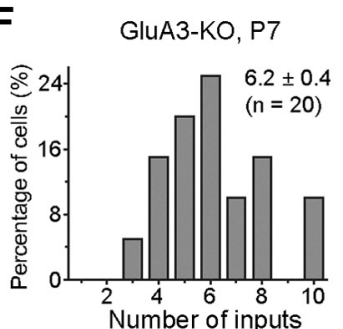

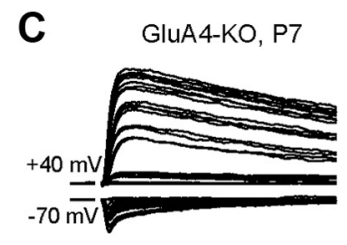
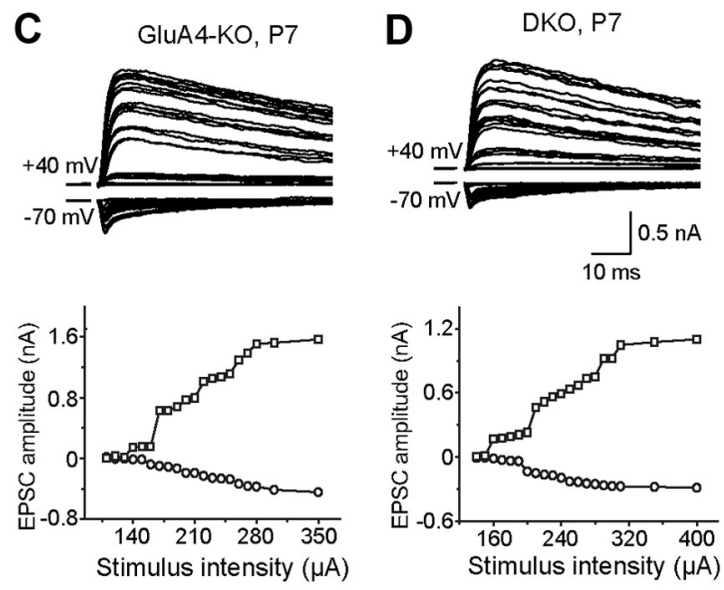

G

GluA4-KO, P7

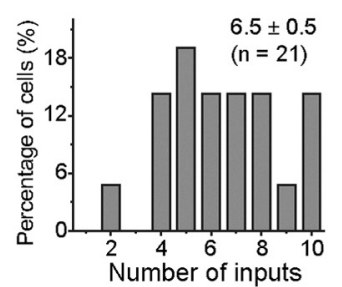

H

DKO, P7

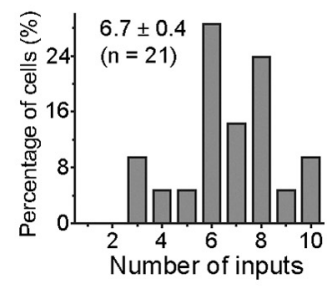

Figure 3. Deletion of GluA3, GluA4, or both did not alter the number of relay inputs in VPm neurons at P7. A through D, upper panels are EPSCs in response to a range of stimulation intensity in P7 neurons; lower panels are the plots of peak amplitudes of EPSCs versus stimulation intensity; the estimated number of inputs are 8, 6, 6, and 7 for WT ( $\boldsymbol{A}$ ), GluA3-K0 (B), GluA4-KO (C) and DKO (D), respectively. $\boldsymbol{E}-\boldsymbol{H}$, The distributions of VPm neurons at P7 receiving different numbers of relay inputs. The average number of fibers per cell for each group was shown in the figures.
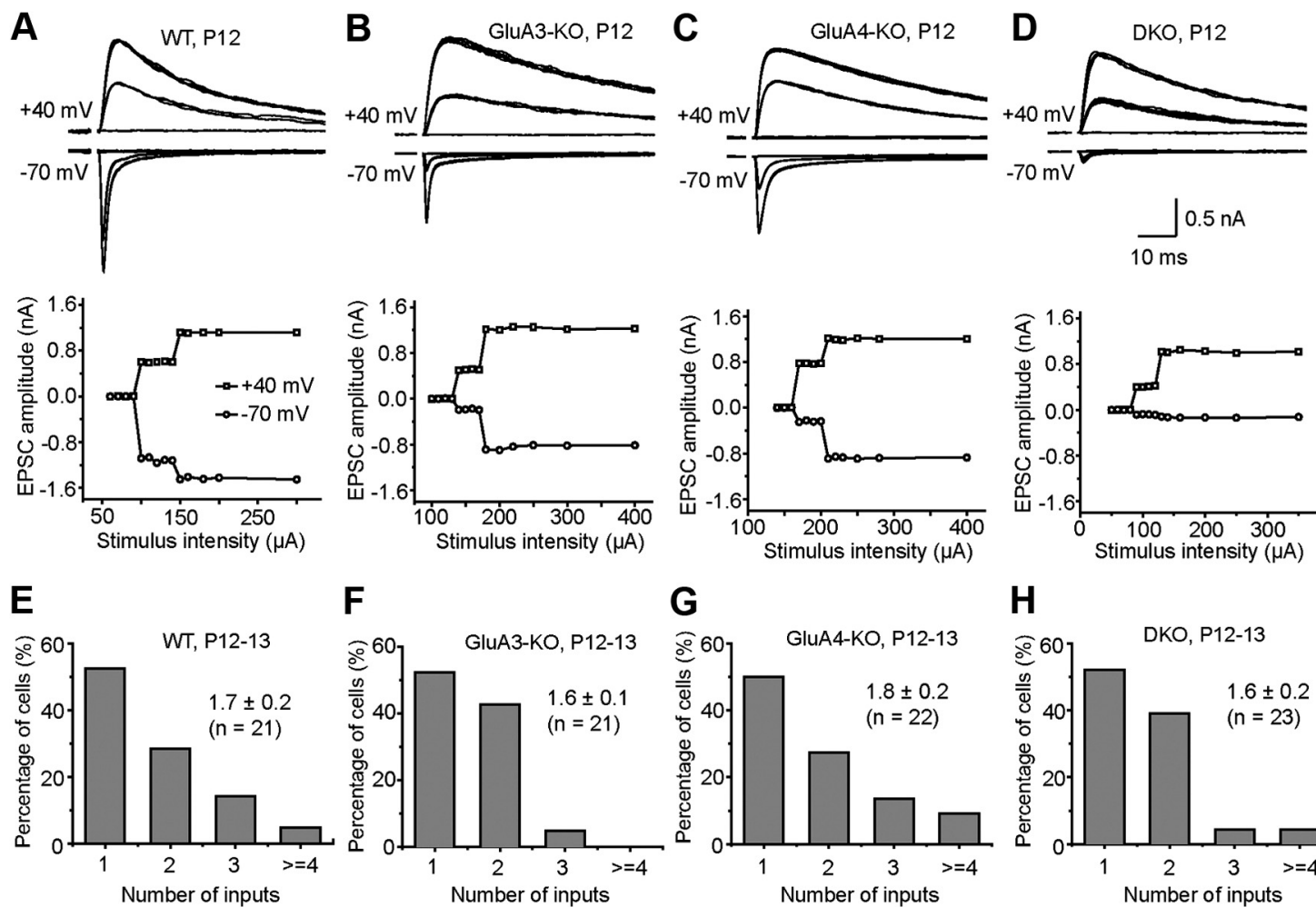

$\mathbf{F}$

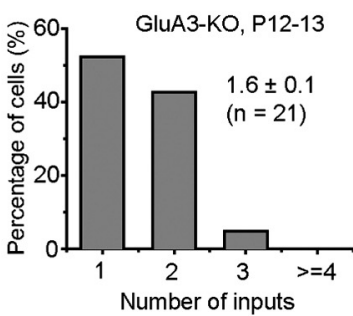

G

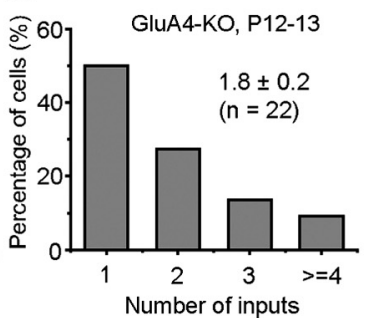

\section{H}

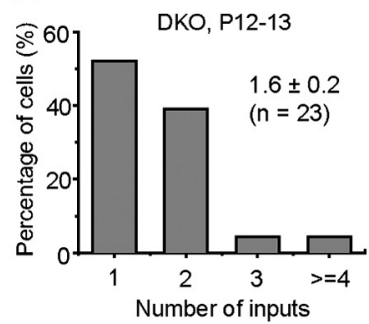

Figure 4. Deletion of GluA3, GluA4, or both did not alter the number of relay inputs in VPm neurons at P12-P13. A-D, Upper panels, EPSCs in response to a range of stimulation intensity in P12 neurons; lower panels are the plots of peak amplitudes of EPSCs versus stimulation intensity; all four cells received two relay inputs. $\boldsymbol{E}-\boldsymbol{H}$, The distributions of VPm neurons at P12-P13 receiving different number of relay inputs. The average number of fibers per cell for each group is shown. 
A
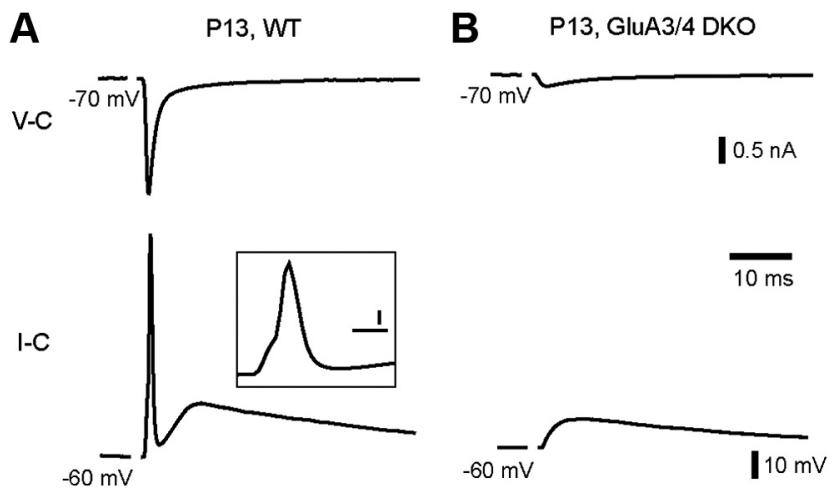

Figure 5. Synaptic responses in VPm neurons of WT and GluA3/4 DK0 mice at P12-P13.A, Upper trace shows EPSC recorded at $-70 \mathrm{mV}$ from a WT neuron at $P 13$, and lower trace shows EPSP and action potential (AP) recorded from the same cell. V-C, Voltage clamp; I-C, current clamp. The inset shows the onset of EPSP with AP. Calibration of the insert: $10 \mathrm{mV}, 1 \mathrm{~ms} . \boldsymbol{B}$, EPSC and EPSP from a GluA3/4 DK0 neuron at P13.

$145.1 \pm 7.2 \mathrm{M} \Omega ; p>0.9)$, or action potential threshold $(-37.3 \pm$ 0.4 vs $-37.8 \pm 0.4 \mathrm{mV} ; p>0.4)$. These results suggest that deletion of both GluA3 and GluA4 may significantly impair synaptic transmission at the lemniscal synapse.

\section{Developmental regulation of GluA3 and GluA4 at the VPm relay synapse}

Expression of AMPAR subunits in the brain is developmentally regulated (Standley et al., 1995; Brennan et al., 1997). We analyzed EPSCs at the VPm relay synapse at P7 from wildtype, GluA3-KO, GluA4-KO, and DKO mice. At P7, the maximal AMPAR-mediated current was significantly reduced in GluA4-KO and DKO mice, but not in GluA3-KO mice (Fig. 6A; $761 \pm 111 \mathrm{pA}, n=19$, for WT; $671 \pm 97 \mathrm{pA}, n=16$, for GluA3$\mathrm{KO}, p>0.5 ; 357 \pm 47 \mathrm{pA}, n=19$, for GluA $4-\mathrm{KO}, p<0.01$ vs WT; $281 \pm 55 \mathrm{pA}, n=19$, for DKO, $p<0.001$ vs WT). Moreover there was no significant difference between GluA4-KO and DKO mice $(p>0.2)$. These results are in contrast with those obtained at P16-P17 when significant differences were found between wildtype and GluA3-KO, and between GluA4-KO and DKO mice (Fig. $1 B$ ).

We also analyzed the maximal EPSC at P12-P13 from wildtype, GluA3-KO, GluA4-KO, and DKO mice. For AMPARmediated responses, the pattern was comparable with that observed at P16-P17. The amplitude of the maximal AMPAREPSC was significant different between DKO and any of the other three groups (Fig. $6 \mathrm{~B} ; 1363 \pm 148 \mathrm{pA}, n=17$ for wild-type; $886 \pm 93 \mathrm{pA}, n=16$ for GluA3-KO; $1074 \pm 85 \mathrm{pA}, n=18$ for GluA4-KO; $364 \pm 51$ pA, $n=22$ for DKO). However, the difference between wild-type and GluA3-KO was not statistically significant at P12-P13 $(p=0.06)$. There was no difference between the four groups in NMDAR-mediated responses $(p>0.5)$.

To further examine the developmental regulation of GluA3 and GluA4 at the synapse, we plotted the maximal responses with age for the four genotypes. As illustrated in Figure 6C, the amplitude of AMPAR-mediated EPSCs increased steadily between P7 and P16 in wild-type (black line; $p<0.001$ ) and GluA4-KO (green line; $p<0.001$ ), with significant increases observed between P7 and P12 ( $p<0.01$ for wild-type; $p<0.001$ for GluA4$\mathrm{KO})$. In contrast, the amplitude of AMPAR-EPSCs in GluA3-KO (red line) or GluA3/4 DKO (blue line) was steady between P7 and P16 $(p>0.2)$. These results indicate that (1) at P7 few GluA3containing AMPARs are present at the VPm relay synapse, and
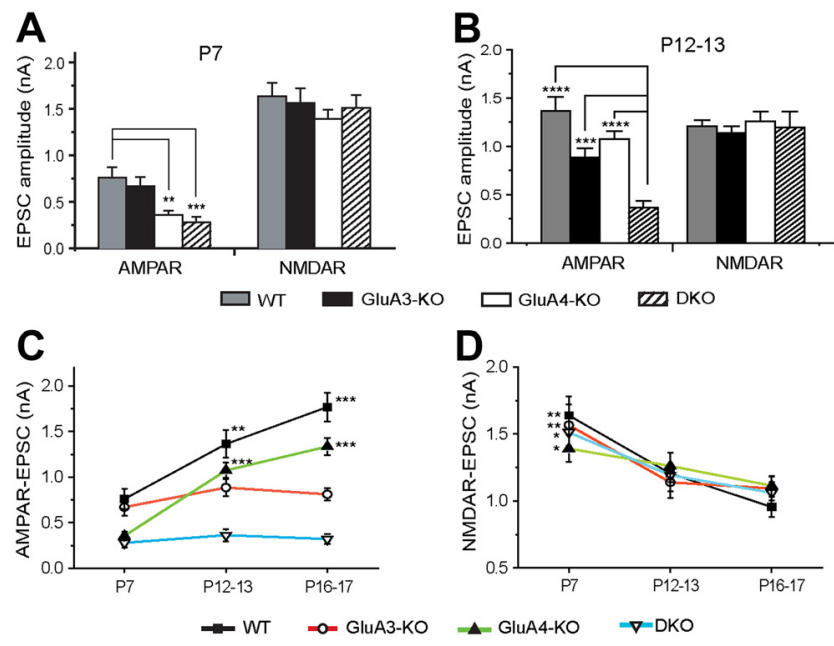

Figure 6. Developmental changes of AMPA receptor composition at the VPm relay synapse $A$, Peak amplitudes of the maximal EPSCs mediated by AMPARs or NMDARs in WT, GluA3-K0, GluA4-K0, and GluA3/4 DKO mice at P7. ${ }^{* * *} p<0.001$ (WT vs DK0), ${ }^{* *} p<0.01$ (WT vs GluA4-K0). At this age, there was no difference in the amplitude of AMPAR-EPSC between WT and GluA3-KO ( $p>0.5$ ), or between GluA4-K0 and GluA3/4 DKO ( $p>0.2)$. B, Peak amplitude of the maximal EPSCs mediated by AMPARs and NMDARs at P12-P13. ${ }^{* * *} p<0.0001$ (DK0 vs WT or GluA3-K0 or GluA4-KO). The differences between WT, GluA3-K0, and GluA4-K0 are not statistically significant. C, Plot of peak amplitudes versus ages for AMPAR-EPSCs. The amplitude of AMPAR-EPSCs in GluA3-KO and DKO did not change between P7 and P16. In WT and GluA4K0, the amplitude of AMPAR-EPSCs increased between P7 and P12-P13 ${ }^{* *} p<0.01$ for WT; ${ }^{* * *} p<0.001$ for GluA4-K0), and between P7 and P16-P17 (*** $\left.p<0.001\right)$. The difference between P12-P13 and P16 - P17 was significant for GluA4-K0 $(p<0.05)$, but not for WT $(p=$ 0.07). D, Plot of peak amplitudes versus ages for NMDAR-EPSCs. For all four groups, the amplitude of NMDAR-EPSCs was significantly reduced between P7 and P16.

GluA4-containing receptors are predominant, and (2) there is a rapid upregulation of GluA3-containing receptors at the synapse during the second week after birth, while the number of GluA4containing receptors remains stable. These findings suggest that developmental strengthening of the lemniscal synapse requires the GluA3 subunit.

Consistent with our previous findings in B6 mice (Arsenault and Zhang, 2006), NMDAR-EPSCs in all four groups showed significant reduction between P7 and P16 (Fig. 6D).

\section{Experience-dependent upregulation of synaptic AMPA receptors requires GluA3}

AMPAR trafficking at glutamatergic synapses is thought to be a key mechanism underlying experience-dependent plasticity in the brain. At the VPm relay synapse of B6 mice, a brief period of sensory deprivation during early life selectively reduces the amplitude of AMPAR-EPSCs (Wang and Zhang, 2008). To determine the roles of GluA3 and GluA4 in this form of synaptic plasticity, we analyzed the effects of sensory deprivation in wildtype, GluA3-KO, and GluA4-KO mice. All large vibrissae on one side of the snort were plucked at $\mathrm{P} 13$, and recordings were made at P14 in slices from both sides of the brain. Consistent with our previous findings (Wang and Zhang, 2008), wild-type VPm neurons on the deprived (contralateral) side showed a $38 \%$ reduction of AMPAR-EPSCs compared with those on the spared (ipsilateral) side (Fig. $7 A, B ; 1033 \pm 134 \mathrm{pA}, n=8$ for deprived; $1663 \pm$ $201 \mathrm{pA}, n=8$ for spared; $p<0.01)$. There was no change in NMDAR-mediated EPSCs $1037 \pm 96$ pA for deprived vs $1107 \pm$ $143 \mathrm{pA}$ for spared; $p>0.7)$. The AMPAR/NMDAR ratio was also reduced in deprived neurons (Fig. $7 B, 1.02 \pm 0.10$ for deprived vs $1.62 \pm 0.20$ for spared; $p<0.05)$. Similar effects were observed in 

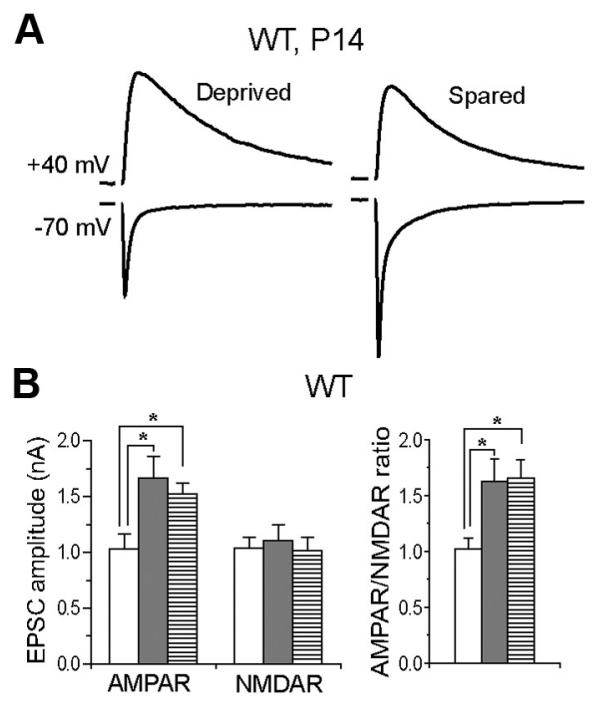

C

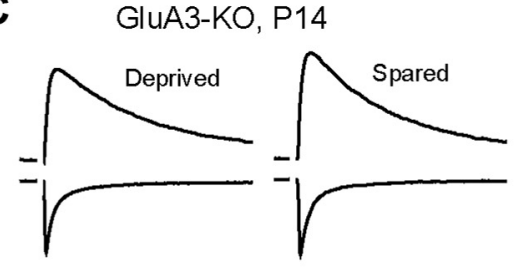

D

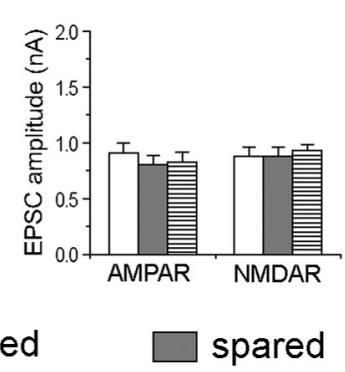

E

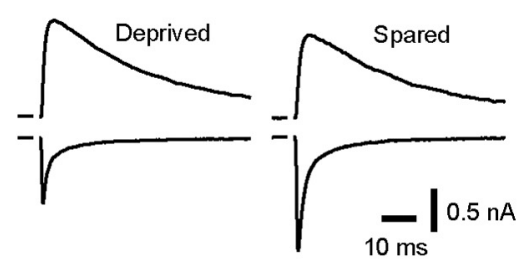

Figure 7. GluA3 is required for experience-dependent plasticity at the VPm relay synapse. $\boldsymbol{A}, \boldsymbol{C}, \boldsymbol{E}$, The maximal EPSCs recorded in deprived (left traces) or spared (right traces) VPm neurons from WT, GluA3-K0, or GluA4-K0 mice at P14. One-side whisker deprivation was done at P13. B, D, F, Left panels show peak amplitudes of the maximal EPSCs mediated by AMPARs or NMDARs recorded from deprived (empty), spared (gray), or untreated (hatched) VPm neurons; right panels show AMPAR/NMDAR ratios of deprived, spared, or untreated VPm neurons. In WT and GluA4-K0, but not GluA3-K0, the amplitude of AMPAR-EPSCs was reduced in deprived neurons. The amplitude of NMDAR-EPSCs showed no change ( $p>0.5)$. For all three groups of mice, there was no difference between spared and untreated neurons.

GluA4-KO mice (Fig. 7E,F): the maximal AMPAR-EPSCs in GluA4-KO mice was $684 \pm 128 \mathrm{pA}(n=10)$ for deprived neurons, and $1153 \pm 119 \mathrm{pA}(n=11)$ for spared neurons $(p<0.01)$. In contrast, whisker deprivation did not alter EPSCs in VPm neurons of GluA3-KO mice (Fig. 7C,D); no change was observed between deprived and spared neurons in AMPAR-EPSCs, NMDAR-EPSCs, or AMPAR/NMDAR ratio ( $p>0.5)$. To examine the possibility that compensation may occur on the spared side, we also recorded nondeprived (untreated) mice at P14. In wild-type, GluA3-KO, and GluA4-KO mice, there was no difference between spared and untreated neurons in AMPAR-EPSC $(p>0.4)$, NMDAR-EPSC $(p>0.5)$, or AMPAR/NMDAR ratio $(p>0.1)$. For untreated neurons, the amplitude of AMPAREPSCs was $1526 \pm 96 \mathrm{pA}(n=10)$ for wild-type, $827 \pm 87 \mathrm{pA}$ $(n=9)$ for GluA3-KO, and $1262 \pm 116 \mathrm{pA}(n=10)$ for GluA4$\mathrm{KO}$; the amplitude of NMDAR-EPSCs was $1016 \pm 121 \mathrm{pA}$ for wild-type, $930 \pm 54 \mathrm{pA}$ for GluA3-KO, and $981 \pm 76 \mathrm{pA}$ for GluA4-KO; AMPAR/NMDA ratio was $1.65 \pm 0.17$ for wild-type, $0.88 \pm 0.06$ for GluA3-KO, and $1.29 \pm 0.07$ for GluA4-KO (Fig. $7 B, D, E$, hatched). In wild-type and GluA4-KO but not GluA3-KO mice, untreated neurons had larger AMPAR-EPSCs and larger AMPAR/NMDAR ratio than deprived neurons.

These results suggest a key role for GluA3 in activitydependent trafficking of AMPARs at the VPm relay synapse. One caveat, however, is that the constitutive knock-out of GluA3 may indirectly alter experience-dependent plasticity at the VPm synapse. We were especially concerned about the possibility that the deletion of GluA3 may dramatically reduce transmission in the $\mathrm{PrV}$. To examine this possibility, we recorded $\mathrm{PrV}$ neurons in acute horizontal slices obtained from wild-type, GluA3-KO, and GluA4-KO mice at P13. Stimulation of the sensory root of the trigeminal nerve evokes monosynaptic EPSCs in PrV neurons (Fig. $8 A, B$ ). The maximal AMPAR- or NMDAR-mediated EPSCs were not significantly different among the three groups (Fig. 8C). The AMPAR/NMDAR ratio was also not different (Fig.
$8 D)$. These results indicate that deletion of GluA3 or GluA4 did not alter the strength of whisker sensory relay synapses in the PrV.

\section{Discussion}

This study demonstrates that synaptic strengthening during development is not necessary for the elimination of redundant synaptic inputs. Deletions of both GluA3 and GluA4 abolished the upregulation of AMPARs at the VPm relay synapse during early life, but had no effect on the elimination of redundant connections during the same period. We also provide evidence that GluA3- and GluA4-containing AMPARs are differentially regulated at the synapse during development, and that GluA3containing receptors are selectively implicated in experiencedependent upregulation of AMPARs at the synapse.

\section{Subunit composition of AMPA receptors at the VPm relay synapse}

Subunit composition controls functional properties, modulation, and trafficking of AMPARs (Malinow and Malenka, 2002; Song and Huganir, 2002). For example, the absence of GluA2 subunit leads to rectification, and an increase in $\mathrm{Ca}^{2+}$ permeability, open probability, and channel conductance (Isaac et al., 2007). Most AMPARs in the brain contain the GluA2 subunit. Recent studies using single-cell gene deletion and patch-clamp recordings have shown that all surface AMPARs in CA1 neurons contain the GluA2 subunit, and that AMPARs at the CA3-CA1 synapse consist predominantly of GluA1-GluA2 heteromers (Lu et al., 2009). These findings are consistent with previous studies using ultrastructural analysis (Wenthold et al., 1996).

The present study demonstrates that AMPARs at the VPm relay synapse have a very different subunit composition than that in CA1 neurons. The majority of AMPARs at the VPm synapse consists of GluA3 and/or GluA4. This is consistent with expression patterns of AMPAR subunits. In adult rats and mice, GluA3 and GluA4 subunits are highly expressed in the VPm, whereas 
GluA1 is not detectable (Liu, 1997; Mineff and Weinberg, 2000). Consistent with our previous study in B6 mice (Wang and Zhang, 2008), the findings of the present studies indicate that the majority of AMPARs at the relay synapse lacks GluA2 subunit. That the amplitude of AMPAREPSCs at $+40 \mathrm{mV}$ did not differ between the four genotypes suggests that neither GluA3 nor GluA4 is associated with GluA2 at the synapse. One caveat is compensation caused by GluA3 or GluA4 knock-out. However, the large reduction of AMPAR-EPSCs at $-70 \mathrm{mV}$ observed in GluA3/4 DKO neurons suggests that compensatory changes of GluA1 or GluA2 are minimal.

\section{Developmental changes in AMPA receptor composition at the VPm relay synapse}

Expression of AMPAR subunits is developmentally regulated in many parts of the brain (Standley et al., 1995; Brennan et al., 1997; Nansen et al., 2000). Little is known, however, about developmental changes in AMPAR composition at the synapse level. Excitatory neurons in the neocortex undergo a development switch from GluA2lacking to GluA2-containing synaptic AMPARs (Kumar et al., 2002; Brill and Huguenard, 2008). Our analyses of AMPAREPSCs in GluA3-KO, GluA4-KO, and GluA3/4 DKO mice during early life revealed a rapid change in AMPAR composition at the VPm relay synapse. At P7, GluA4 and possibly GluA2 are the major subunits, and few receptors contain GluA3. In contrast, GluA3 becomes the major subunit at P16-P17. Furthermore, by comparing AMPAR-EPSCs at P7 with those at P16-P17, we found that the number of GluA4-containing receptors remains unchanged between P7 and P16. Thus, VPm relay synapses acquire GluA4-containing receptors at early stages of synapse development, and this is followed by a surge in GluA3-containing receptors.

\section{Activity-dependent regulation of AMPA receptors at the VPm relay synapse}

Activity-dependent changes in synaptic strength are a key mechanism for synaptic plasticity during development and in adults. At glutamatergic synapses in the brain, activity-dependent trafficking of AMPARs is a major mechanism underlying synaptic plasticity (Malinow and Malenka, 2002; Song and Huganir, 2002). A large number of studies have shown that activitydependent trafficking of GluA1- and GluA2-containing receptors is important for long-term potentiation and long-term depression (Cull-Candy et al., 2006; Isaac et al., 2007; Kerchner and Nicoll, 2008). Little is known, however, about the trafficking of GluA3- or GluA4-containing receptors in vivo. At the VPm relay synapse, the rapid surge in GluA3-containing receptors during the second week raised the possibility that trafficking of GluA3-containing receptors is strongly regulated by activity. Consistent with this hypothesis, we found that experiencedependent changes in AMPAR-mediated EPSCs were absent in GluA3-KO mice, but normal in GluA4-KO mice (Fig. 7). In addition, the early presence of GluA4-containing receptors in VPm
B
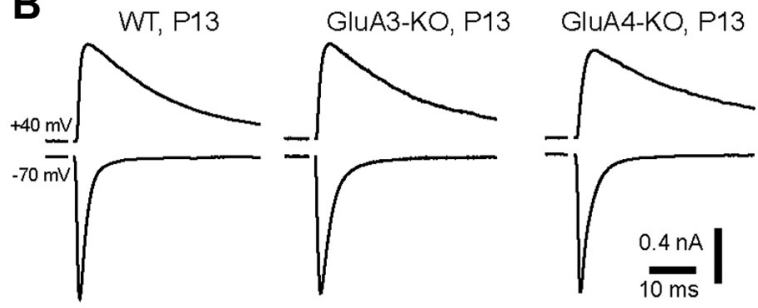

D

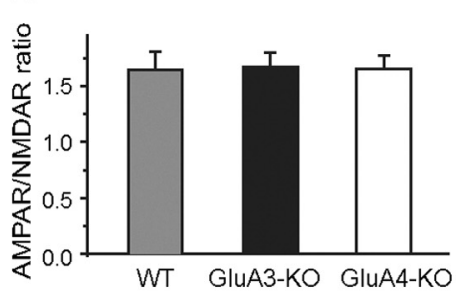

Figure 8. Excitatory synaptic responses at whisker relay synapses in PrV neurons of WT, GluA3-K0, and GluA4-KO mice. $\boldsymbol{A}, \mathrm{A}$ che slice preparation. Horizontal slices through the brainstem were cut at $250 \mu \mathrm{m}$ in thickness. Whole-cell 作 $88 \mathrm{pA}(n=19)$ for WT, $831 \pm 98 \mathrm{pA}(n=11)$ for GluA3-K0, and $861 \pm 79 \mathrm{pA}(n=9)$ for GluA4-KO $(p>0.2)$. The mean $\mathrm{PA}(n=9)$ for GluA4-KO $(p>0.3)$. D, The AMPAR/NMDAR ratio was $1.64 \pm 0.17$ for WT, $1.67 \pm 0.13$ for GluA3-K0, and $1.65 \pm$

neurons suggests that spontaneous activity may be sufficient for membrane trafficking of GluA4-containing receptors, consistent with studies using overexpression of GluA4 in cultured neurons (Zhu et al., 2000).

It remains to be determined how sensory experience selectively regulates GluA3-containing receptors at this synapse. Expression of GluA3 increases in the VPm during the second week after birth (Allen Brain Atlas: http://developingmouse.brainmap.org/). Thus early sensory experience may promote the transcription of GluA3 in the VPm. However, the reduction in AMPAR-EPSCs by sensory deprivation was observed within $24 \mathrm{~h}$ (Fig. 7). It is possible, therefore, that sensory experience promotes the trafficking of GluA3-containing AMPARs. As other members of AMPAR subunits, GluA3 can be phosphorylated at the C-terminal (Song and Huganir, 2002). Future studies are needed to determine the role of GluA3 phosphorylation in receptor trafficking.

\section{Removal of redundant inputs is independent of synaptic strengthening}

In the developing nervous systems, strengthening of immature synapses often precedes the removal of redundant inputs (Colman et al., 1997; Hashimoto and Kano, 2003). It was unclear, however, whether these two components of synaptic refinement are mechanistically related. In the hippocampus, deletion of all AMPARs had no effect on dendritic arborization or spine density of CA1 neurons (Lu et al., 2009), although the number of presynaptic inputs was not determined in the study. In the PrV-VPm pathway, elimination of redundant inputs during the second week is accompanied by a large increase in AMPAR-mediated EPSCs and a decrease in NMDAR-mediated EPSCs, with little change in presynaptic release probability (Arsenault and Zhang, 2006). Thus, upregulation of synaptic AMPARs is the key mechanism underlying synapse strengthening in this pathway. Sur- 
prisingly, deletions of both GluA3 and GluA4, which disrupt transmission at VPm relay synapses, had no effect on the pruning process. In slices obtained from P12-P13 mice, stimulation of PrV axons consistently evoked action potentials in VPm neurons in wild-type animals, but failed to do so in $80 \%$ of VPm neurons in GluA3/4 DKO animals. It should be pointed out that synaptic transmission detected in slices may be significantly different from that that occurs in vivo. Unlike neurons in vivo, which oscillate between a depolarized up state and a hyperpolarized down state, thalamic neurons in slices show only the down state. Another factor is the loss of neuromodulation in slices. Both factors can lead an overestimation of failure rate of synaptic transmission. In vivo recording is needed to ascertain the effect of GluA3/4 deletions on synaptic transmission in VPm neurons in P12-P13 mice.

In GluA3/4 DKO mice, the remaining AMPARs in VPm neurons appear to contain the GluA2 subunit. These GluA2containing receptors make up a small fraction of AMPARs at the VPm relay synapse in wild-type neurons, and do not contribute to the upregulation of AMPARs during the second week. However, we cannot rule out the possibility that the GluA2 subunit plays a role in synaptic refinement. GluA2 is highly expressed in the thalamus during embryonic development and in newborns (Allen Brain Atlas: http://developingmouse.brain-map.org/); the majority of GluA2-containing receptors at these ages are likely to be extrasynaptic. These GluA2-containing AMPARs could have an important role in early stages of synapse development.

Our previous study showed that sensory deprivation by plucking whiskers at P12-P13 disrupted both elimination and strengthening of thalamic relay synapses (Wang and Zhang, 2008). The results of the present study indicate a dissociation of these two processes. Deletion of GluA3 abolished experiencedependent strengthening of relay synapses without any effect on synapse elimination. A major difference between this and our previous study is how activity of the whisker sensory pathway was altered. Our previous study used whisker deprivation that reduces synaptic transmission in both the PrV and VPm; in this study, synaptic transmission in GluA3-KO mice was affected in the VPm but not PrV. Therefore unlike whisker-deprived mice, the activity level of PrV neurons was probably normal in GluA3-KO mice. The dissociation of elimination and experiencedependent strengthening of relay synapses in GluA3-KO mice raises two possibilities: (1) signaling through NMDA and the remaining AMPA receptors in VPm neurons may be sufficient for elimination of relay synapses; and (2) normal activity of PrV neurons, but not synaptic transmission in the VPm, is required for elimination of relay inputs.

Our findings demonstrate that synaptic strengthening is not required for the elimination of redundant inputs. However, it should be noted that our experiments did not test the hypothesis of activity-dependent competition. All PrV inputs to VPm neurons were weakened in GluA3/4 DKO mice. It has been shown that at the neuromuscular junction, activity-dependent competition plays a crucial role in pruning even when synaptic transmission is significantly reduced for all synapses (Buffelli et al., 2003). In the case of the PrV-VPm pathway, it is unclear whether immature inputs compete for their ability to fire postsynaptic neurons. Signaling through glutamate receptors may be sufficient to mediate activity-dependent competition.

\section{References}

An MC, Lin W, Yang J, Dominguez B, Padgett D, Sugiura Y, Aryal P, Gould TW, Oppenheim RW, Hester ME, Kaspar BK, Ko CP, Lee KF (2010)
Acetylcholine negatively regulates development of the neuromuscular junction through distinct cellular mechanisms. Proc Natl Acad Sci U S A 107:10702-10707.

Arsenault D, Zhang ZW (2006) Developmental remodelling of the lemniscal synapse in the ventral basal thalamus of the mouse. J Physiol 573:121-132.

Beyer B, Deleuze C, Letts VA, Mahaffey CL, Boumil RM, Lew TA, Huguenard JR, Frankel WN (2008) Absence seizures in $\mathrm{C} 3 \mathrm{H} / \mathrm{HeJ}$ and knockout mice caused by mutation of the AMPA receptor subunit Gria4. Hum Mol Genet 17:1738-1749.

Bredt DS, Nicoll RA (2003) AMPA receptor trafficking at excitatory synapses. Neuron 40:361-379.

Brennan EM, Martin LJ, Johnston MV, Blue ME (1997) Ontogeny of nonNMDA glutamate receptors in rat barrel field cortex: II. Alpha-AMPA and kainate receptors. J Comp Neurol 386:29-45.

Brill J, Huguenard JR (2008) Sequential changes in AMPA receptor targeting in the developing neocortical excitatory circuit. J Neurosci 28:13918-13928.

Buffelli M, Burgess RW, Feng G, Lobe CG, Lichtman JW, Sanes JR (2003) Genetic evidence that relative synaptic efficacy biases the outcome of synaptic competition. Nature 424:430-434.

Chen C, Regehr WG (2000) Developmental remodeling of the retinogeniculate synapse. Neuron 28:955-966.

Collingridge GL, Isaac JT, Wang YT (2004) Receptor trafficking and synaptic plasticity. Nat Rev Neurosci 5:952-962.

Colman H, Nabekura J, Lichtman JW (1997) Alterations in synaptic strength preceding axon withdrawal. Science 275:356-361.

Cull-Candy S, Kelly L, Farrant M (2006) Regulation of Ca2+-permeable AMPA receptors: synaptic plasticity and beyond. Curr Opin Neurobiol 16:288-297.

Hashimoto K, Kano M (2003) Functional differentiation of multiple climbing fiber inputs during synapse elimination in the developing cerebellum. Neuron 38:785-796.

Isaac JT, Nicoll RA, Malenka RC (1995) Evidence for silent synapses: implications for the expression of LTP. Neuron 15:427-434.

Isaac JT, Crair MC, Nicoll RA, Malenka RC (1997) Silent synapses during development of thalamocortical inputs. Neuron 18:269-280.

Isaac JT, Ashby MC, McBain CJ (2007) The role of the GluR2 subunit in AMPA receptor function and synaptic plasticity. Neuron 54:859-871.

Kano M, Hashimoto K (2009) Synapse elimination in the central nervous system. Curr Opin Neurobiol 19:154-161.

Kerchner GA, Nicoll RA (2008) Silent synapses and the emergence of a postsynaptic mechanism for LTP. Nat Rev Neurosci 9:813-825.

Kumar SS, Bacci A, Kharazia V, Huguenard JR (2002) A developmental switch of AMPA receptor subunits in neocortical pyramidal neurons. J Neurosci 22:3005-3015.

Lichtman JW, Colman H (2000) Synapse elimination and indelible memory. Neuron 25:269-278.

Liu XB (1997) Subcellular distribution of AMPA and NMDA receptor subunit immunoreactivity in ventral posterior and reticular nuclei of rat and cat thalamus. J Comp Neurol 388:587-602.

Lu W, Shi Y, Jackson AC, Bjorgan K, During MJ, Sprengel R, Seeburg PH, Nicoll RA (2009) Subunit composition of synaptic AMPA receptors revealed by a single-cell genetic approach. Neuron 62:254-268.

Luo L, O'Leary DD (2005) Axon retraction and degeneration in development and disease. Annu Rev Neurosci 28:127-156.

Malinow R, Malenka RC (2002) AMPA receptor trafficking and synaptic plasticity. Annu Rev Neurosci 25:103-126.

Mayer ML, Armstrong N (2004) Structure and function of glutamate receptor ion channels. Annu Rev Physiol 66:161-181.

Mineff EM, Weinberg RJ (2000) Differential synaptic distribution of AMPA receptor subunits in the ventral posterior and reticular thalamic nuclei of the rat. Neuroscience 101:969-982.

Misgeld T, Burgess RW, Lewis RM, Cunningham JM, Lichtman JW, Sanes JR (2002) Roles of neurotransmitter in synapse formation: development of neuromuscular junctions lacking choline acetyltransferase. Neuron 36:635-648.

Nansen EA, Jokel ES, Lobo MK, Micevych PE, Ariano MA, Levine MS (2000) Striatal ionotropic glutamate receptor ontogeny in the rat. Dev Neurosci 22:329-340.

Petralia RS, Esteban JA, Wang YX, Partridge JG, Zhao HM, Wenthold RJ, Malinow R (1999) Selective acquisition of AMPA receptors over post- 
natal development suggests a molecular basis for silent synapses. Nat Neurosci 2:31-36.

Sanes JR, Lichtman JW (1999) Development of the vertebrate neuromuscular junction. Annu Rev Neurosci 22:389-442.

Song I, Huganir RL (2002) Regulation of AMPA receptors during synaptic plasticity. Trends Neurosci 25:578-588.

Standley S, Tocco G, Tourigny MF, Massicotte G, Thompson RF, Baudry M (1995) Developmental changes in alpha-amino-3-hydroxy-5-methyl-4isoxazole propionate receptor properties and expression in the rat hippocampal formation. Neuroscience 67:881-892.

Timofeeva E, Dufresne C, Sík A, Zhang ZW, Deschênes M (2005) Cholinergic modulation of vibrissal receptive fields in trigeminal nuclei. J Neurosci 25:9135-9143.

Wang H, Zhang ZW (2008) A critical window for experience-dependent plasticity at whisker sensory relay synapse in the thalamus. J Neurosci 28:13621-13628

Wenthold RJ, Petralia RS, Blahos J II, Niedzielski AS (1996) Evidence for multiple AMPA receptor complexes in hippocampal CA1/CA2 neurons. J Neurosci 16:1982-1989.

Wu G, Malinow R, Cline HT (1996) Maturation of a central glutamatergic synapse. Science 274:972-976.

Zhang ZW (2004) Maturation of layer V pyramidal neurons in the rat prefrontal cortex: intrinsic properties and synaptic function. J Neurophysiol 91:1171-1182.

Zhang ZW (2006) Developmental refinement in the mammalian thalamus. Crit Rev Neurobiol 18:49-59.

Zhu JJ, Esteban JA, Hayashi Y, Malinow R (2000) Postnatal synaptic potentiation: delivery of GluR4-containing AMPA receptors by spontaneous activity. Nat Neurosci 3:1098-1106. 